\title{
A new enniatin antibiotic from the endophyte Fusarium tricinctum Corda
}

\author{
Ahmed M Zaher ${ }^{1,2}$, Makboul A Makboul ${ }^{2}$, Ahmad M Moharram³ ${ }^{3}$ Babu L Tekwani ${ }^{4}$ and Angela I Calderón ${ }^{1}$ \\ Enniatins (ENs), a group of antibiotics commonly produced by various strains of Fusarium, are six-membered cyclic \\ depsipeptides formed by the union of three molecules of $\mathrm{D}$ - $\alpha$-hydroxyisovaleric acid and three $\mathrm{N}$-methyl-L-amino acids. The \\ endophyte Fusarium tricinctum Corda was isolated from the fruits of Hordeum sativum Jess. and cultivated on a rice medium. \\ The fungal metabolites were extracted with methanol and were identified, employing liquid chromatography-mass spectrometry \\ as ENs A, A1, B, B1, B2 and Q. EN Q is a new analog of EN A and the occurrence of EN B2 is reported for the first time from \\ this endophyte, in addition to four well-known ENs (A, A1, B and B1). The methanol extract of $F$. tricinctum showed mild \\ antibacterial and antileishmanial activities. Additionally the tested extract displayed inhibition of the activity of thioredoxin \\ reductase enzyme of Plasmodium falciparum.
}

The Journal of Antibiotics (2015) 68, 197-200; doi:10.1038/ja.2014.129; published online 15 October 2014

\section{INTRODUCTION}

Enniatins (ENs) are antibiotics produced by members of Fusarium, Halosarpheia, Verticillium and Alternaria. ${ }^{1,2}$ The ENs are biosynthesized from three molecules of $\mathrm{N}$-methyl-L-amino acids and three molecules of $\mathrm{D}$ - $\alpha$-hydroxyisovaleric acid to form an 18 -membered cyclic depsipeptide. ${ }^{3}$ ENs exhibit their antimicrobial, herbicidal and insecticidal activities ${ }^{4}$ by disrupting the ion selectivity of the cell walls. ${ }^{5}$ They are capable of forming stoichiometric complexes with various cations facilitating the transport of the latter through biological membranes (especially the mitochondrial membranes resulting in uncoupling of oxidative phosphorylation). ${ }^{6}$ Another reported mechanism for the insecticidal activity of ENs involves their capacity to inhibit the acyl-CoA:cholesterol acyltransferase activity. ${ }^{7}$ The mechanism of action of antiplasmodial and antimycobacterial activities of ENs is still awaiting clarification. ${ }^{1}$ The already characterized ENs are A, A1, B and B1. ${ }^{8,9}$ Other reported EN analogs include B2, B3, B4, D, E, F, G, H, I and $1688 \mathrm{MK}^{7,10,11}$

In the present study, Fusarium tricinctum Corda was isolated from the fruits of Hordeum sativum Jess. and subsequently cultivated on a rice medium. The methanol extract of the endophyte was analyzed by liquid chromatography-mass spectrometry (LC-MS) and liquid chromatography-tandem mass spectrometry (LC-MS/MS), which resulted in the characterization of six EN antibiotics (A, A1, B, B1, $\mathrm{B} 2$ and $\mathrm{Q})$. In addition to the five known ENs (A, A1, B, B1 and B2), ${ }^{12}$ a new analog of EN A was identified and designated as EN Q. It is noteworthy that the EN B2 has not been previously reported from the fungus under study.

\section{MATERIALS AND METHODS}

Chemicals and growth media

All organic solvents were of high-performance liquid chromatography grade and were purchased from Thermo Fisher (Hanover Park, IL, USA). Purified water was prepared using a Milli-Q purification system (Millipore, Billerica, MA, USA). Rice medium was prepared by overnight maceration of $100-\mathrm{g}$ white rice in $100 \mathrm{ml}$ of water in a one liter Erlenmeyer flask, before autoclaving at $1.055 \mathrm{kgf} \mathrm{cm}^{-2}$ for $30 \mathrm{~min}$.

\section{Fungal culture and extraction of secondary metabolites}

The endophyte F. tricinctum was isolated from the fruits of H. sativum Jess. and grown on three 1000-ml Erlenmeyer flasks each containing $100 \mathrm{~g}$ of rice that were soaked overnight in distilled water $(100 \mathrm{ml})$ before being autoclaved at $1.055 \mathrm{kgf} \mathrm{cm}^{-2}$ for $30 \mathrm{~min}$. After the flasks had cooled down to room temperature, they were inoculated with hyphal tips of the fungus and left to grow in the incubator for three weeks at $25^{\circ} \mathrm{C}$. Following incubation, the fermented rice substrate in each flask was first homogenized with a homogenizer (Bee International, South Easton, MA, USA) and then extracted three times with $200 \mathrm{ml}$ of methanol each time. The combined methanol extracts were filtered through Whatman No.1 filter paper (GE Healthcare Bio-Sciences, Pittsburgh, PA, USA) and then concentrated using a rotary evaporator (Buchi, Flawil, Switzerland) and fully dried using nitrogen stream (Organomation Associate, Berlin, MA, USA). The dried extract was stored at $-20^{\circ} \mathrm{C}$ until analyzed by LC-MS.

\section{LC-MS and LC-MS/MS analyses}

The LC separation of the fungal secondary metabolites was achieved employing a ZORBAX Eclipse XDB- $\mathrm{C}_{18}$ column $(5 \mu \mathrm{m}, 4.6 \times 150 \mathrm{~mm}$ ) (Agilent Technologies, New Castle, DE, USA). Elution of the metabolites was carried out

\footnotetext{
${ }^{1}$ Department of Drug Discovery and Development, Harrison School of Pharmacy, Auburn University, Auburn, AL, USA; ${ }^{2}$ Department of Pharmacognosy, Faculty of Pharmacy, Assiut University, Assiut, Egypt; ${ }^{3}$ Mycology Center, Faculty of Science, Assiut University, Assiut, Egypt and ${ }^{4}$ National Center for Natural Products Research, School of Pharmacy, University of Mississippi, Oxford, MS, USA

Correspondence: Dr Al Calderón, Department of Drug Discovery and Development, Harrison School of Pharmacy, Auburn University, 4306 Walker Building, Auburn, AL 36849, USA.

E-mail: aic0001@auburn.edu

Received 6 June 2014; revised 15 August 2014; accepted 22 August 2014; published online 15 October 2014
} 
using a gradient mobile phase consisting of (A) $0.1 \%$ formic acid in water and (B) methanol at $25^{\circ} \mathrm{C}$. The flow rate was $0.6 \mathrm{ml} \mathrm{min}{ }^{-1}$. A linear gradient was run as follows: $0 \mathrm{~min}, 0 \% \mathrm{~B} ; 6 \mathrm{~min}, 10 \% \mathrm{~B} ; 8 \mathrm{~min}, 60 \% \mathrm{~B} ; 40 \mathrm{~min}, 100 \% \mathrm{~B}$; $42 \mathrm{~min}, 0 \% \mathrm{~B}$. A total of $5 \mathrm{mg}$ of methanol extract was dissolved in $1 \mathrm{ml}$ methanol and then diluted 1:60. Sample injection volume was $0.001 \mathrm{ml}$ at a concentration of $0.083 \mathrm{mg} \mathrm{ml}^{-1}$. The gradient was started immediately after the sample injection. Detection of compounds was achieved by using an Agilent 6520 quadrupole-time-of-flight (Q-TOF) mass spectrometer equipped with a 1220 rapid resolution liquid chromatography (RRLC) system and an electrospray ion source (Agilent Technologies, Little Falls, DE, USA) was used for fingerprinting and chemical profiling of the fungal extract.

\section{Evaluation of antibacterial, antifungal, antileishmanial and} antimalarial activities

All conditions for the antibacterial, antifungal, antileishmanial and antimalarial screening tests were the same as those previously published methods. ${ }^{13}$ The antibacterial activity of the methanol extract was tested against Gram-positive bacteria methicillin-resistant Staphylococcus aureus ATCC 12228 and Mycobacterium intracellulare ATCC 13950, and Gram-negative bacteria E coli ATCC 25922 and Pseudomonas aeruginosa ATCC 1045, and the antifungal activity was tested against Candida albicans ATCC 10231, C. glabrata ATCC 90030, C. krusei
ATCC 14243, Aspergillus fumigatus ATCC 1022 and Cryptococcus neoformans ATCC MYA-4567. Alamar Blue (Laboserv, Giessen, Germany) assay was used to determine the antileishmanial activity of the methanol extract against Leishmania donovani ATCC 39930D. The antimalarial activity was assessed by testing the extract against Plasmodium falciparum D6. The antibacterial, antifungal and antiparasitic activities were reported as $\mathrm{IC}_{50}$ and $\mathrm{IC}_{90}$ values. Extracts which display $\mathrm{IC}_{50}$ values $<10 \mu \mathrm{g} \mathrm{ml}^{-1}$ were considered active.

Based on reports in the literature about the characteristic antibacterial activity of the compound class ENs, ${ }^{2,3,8}$ the antibacterial activity was retested against Staphylococcus aureus Xen 31 (methicillin-resistant Staphylococcus aureus) (PerkinElmer, Waltham, MA, USA) in an Agar disc diffusion method for antibacterial screening ${ }^{14}$ at the amounts of 0.06 and $0.15 \mathrm{mg}$. Antibacterial activity of extracts was categorized according to the size of the zone of inhibition ( $>12 \mathrm{~mm}$, highly active; $>9$ and $<12 \mathrm{~mm}$, moderately active; $<12 \mathrm{~mm}$, inactive).

\section{LC-MS-based screening PfTrxR inhibitor assay}

All chemicals, reagents, sample preparation and LC-MS analytical conditions used in the $P$. falciparum thioredoxin reductase (PfTrxR) inhibitory activity assay were the same as those of the previously published methods. ${ }^{15}$ The methanol extract of the endophyte at $0.01 \mathrm{mg} \mathrm{ml}^{-1}$ in $1 \%$ dimethyl sulfoxide

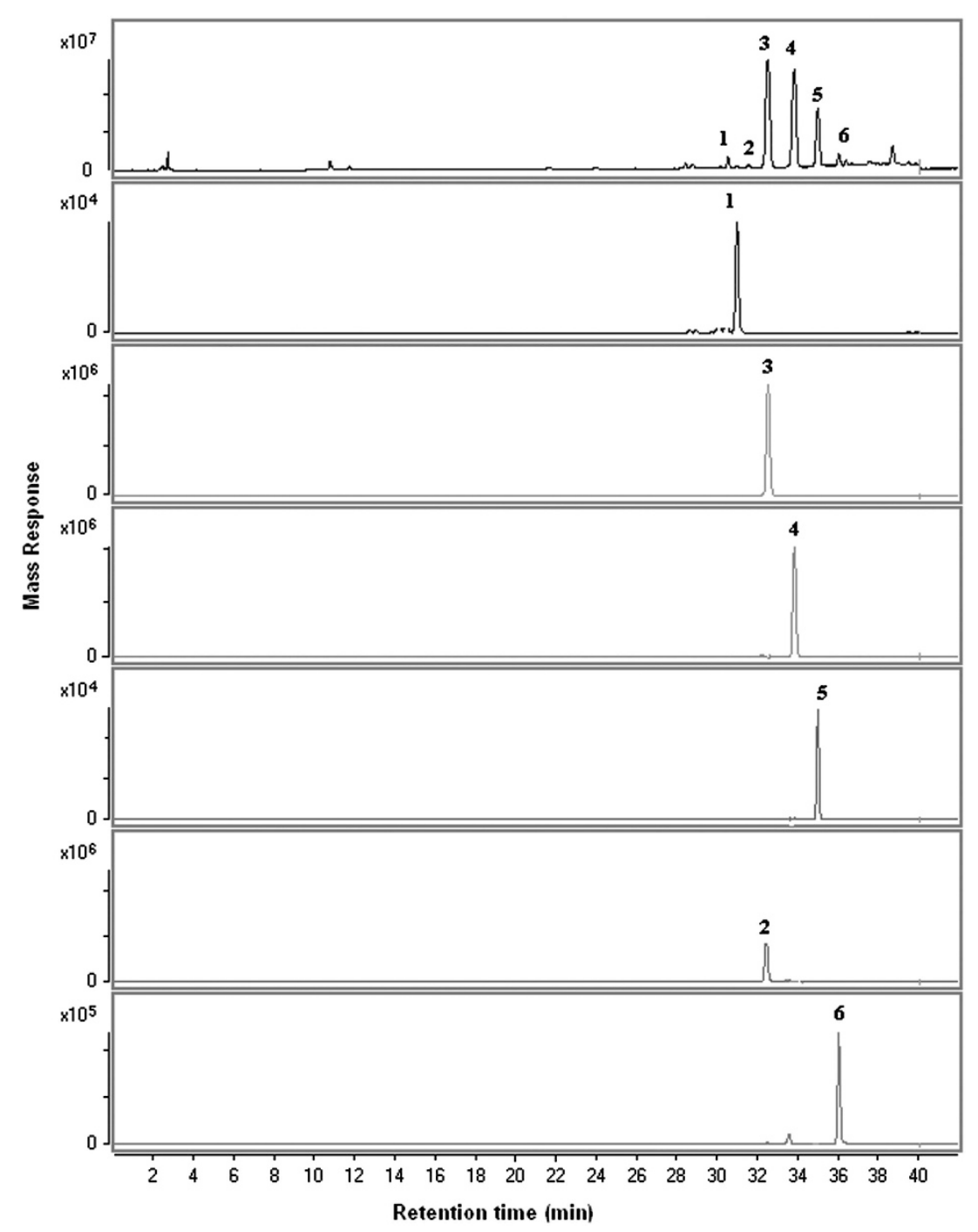

Figure 1 Extractive ion chromatogram of methanol extract of $F$. tricinctum acquired in (+)-electrospray liquid chromatography and mass spectrometry. A full color version of this figure is available at The Journal of Antibiotics journal online. 
was incubated with $0.5 \mathrm{~mm}$ PfTrxR enzyme and $200 \mathrm{~mm}$ NADPH in the assay buffer (PE buffer: $100 \mathrm{~mm}$ potassium phosphate and $2 \mathrm{~mm}$ EDTA, $\mathrm{pH}$ 7.4). The reaction of enzyme was initiated by adding $2.5 \mathrm{~mm} \operatorname{Trx}-\mathrm{S}_{2}$ and incubated at $25^{\circ} \mathrm{C}$ for $30 \mathrm{~min}$. The reaction was stopped by addition of $0.1 \%$ formic acid. The reaction mixture was filtered through a $30-\mathrm{kDa}$ molecular weight cut-off ultra-filtration membrane after centrifugation at 13000 r.p.m. at $20^{\circ} \mathrm{C}$ for $10 \mathrm{~min}$. The filtrate including the reaction product $\left(\mathrm{Trx}-(\mathrm{SH})_{2}\right)$ was analyzed by LC-MS. A control experiment (without inhibitor) was performed the same way and the amount of thioredoxin dithiol $\left(\operatorname{Trx}-(\mathrm{SH})_{2}\right)$ which is the end product of Pf TrxR enzyme was used to reference the activity in the test sample of the methanol extract. The reference compound was bis-2,4-dinitrophenyl sulfide with an $\mathrm{IC}_{50}$ value of $0.52 \mu \mathrm{m}$.

\section{RESULTS}

Identification of compounds by positive ESI-LC-MS

By using $0.1 \%$ formic acid in water as solvent (A) and methanol as solvent (B) in a gradient elution as mobile phase and $\mathrm{C}_{18}$ reversed phase as stationary phase, with elution time $42 \mathrm{~min}$ and in a (+) electrospray ion mode, we obtained six chromatographic peaks as shown in Figure 1 and summarized in Table 1. The identification of six compounds (Figure 2) in the methanol extract was performed by LC-MS based on accurate mass measurement, molecular formula, double bond equivalent, tandem mass spectrometry fragmentation and was confirmed by comparison with the previously available MS data of the compounds under study. ${ }^{11,12,16}$

The ENs that were detected in the methanol extract of $F$. tricinctum have the same basic skeleton with different substituted groups $R_{1-6}$ (Figure 2). They have the same tandem mass spectrometry fragmentation pattern characterized by the cleavage of amide and ester bonds (Table 1 and Figure 3). The observed fragmentations agreed with those previously published in the literature for $\mathrm{ENs} \mathrm{A}, \mathrm{A} 1, \mathrm{~B}$ and $\mathrm{B} 1^{11}$, and B2. ${ }^{16}$

\section{Biological activity}

Antibacterial, antifungal, antimalarial and antileishmanial activities. The antimicrobial activities of the methanol extract of the fungus F. tricinctum containing six ENs (A, A1, B, B1, B2 and Q) tested against Gram-positive and Gram-negative bacteria and fungi, and antimalarial activity yielded $\mathrm{IC}_{50}$ values of $>10 \mu \mathrm{g} \mathrm{ml}^{-1}$. Antileishmanial activity of the methanol extract against $L$. donovani was determined as mild with $\mathrm{IC}_{50}$ values closer to the threshold 10 $\mu \mathrm{g} \mathrm{ml}{ }^{-1}$. The restest of the extract against Staphylococcus aureus Xen 31 (methicillin-resistant Staphylococcus aureus) showed mild activity with inhibition zones within the threshold of activity.

\section{PfTrxR inhibitory activity}

The methanol extract of $F$. tricinctum showed inhibition of the activity of PfTrxR enzyme by a factor of $95 \%$ at $100 \mu \mathrm{g} \mathrm{ml}^{-1}$. From these results and the presence of the antimalarial enniantin $\mathrm{B}$ against $P$. falciparum $\mathrm{K} 1,{ }^{1}$ we concluded that the mechanism of action might be due to inhibition of PfTrxR enzyme.

\section{DISCUSSION}

LC-MS and LC-MS/MS analyses of the methanol extract of the endophytic fungus $F$. tricinctum led to the identification of six EN antibiotics (A, A1, B, B1, B2 and Q). This study reports EN Q as a new $\mathrm{EN}$ analog and EN B2 is reported here for the first time in the endophyte and their identification was based on the characteristic MS fragmentation (Figure 3). The methanol extract of ENs showed mild antibacterial activity. The methanol extract of F. tricinctum showed inhibition of PfTrxR enzymatic activity. The methanol extract showed also mild antileishmanial activity against $L$. donovani ATTC $39930 \mathrm{D}$ with the $\mathrm{IC}_{50}$ value $=16.96 \mu \mathrm{g} \mathrm{ml}^{-1}$ and the $\mathrm{IC}_{90}$ value $=30.4 \mu \mathrm{g} \mathrm{ml}^{-1}$.

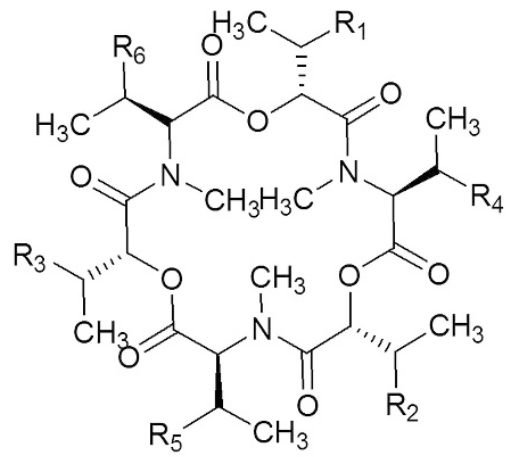

\begin{tabular}{lllllll}
\hline Enriatin & $\mathrm{R}_{1}$ & $\mathrm{R}_{2}$ & $\mathrm{R}_{3}$ & $\mathrm{R}_{4}$ & $\mathrm{R}_{5}$ & $\mathrm{R}_{6}$ \\
\hline B & $\mathrm{CH}_{3}$ & $\mathrm{CH}_{3}$ & $\mathrm{CH}_{3}$ & $\mathrm{CH}_{3}$ & $\mathrm{CH}_{3}$ & $\mathrm{CH}_{3}$ \\
\hline B1 & $\mathrm{CH}_{3}$ & $\mathrm{CH}_{3}$ & $\mathrm{CH}_{3}$ & $\mathrm{CH}_{2} \mathrm{CH}_{3}$ & $\mathrm{CH}_{3}$ & $\mathrm{CH}_{3}$ \\
\hline A1 & $\mathrm{CH}_{3}$ & $\mathrm{CH}_{3}$ & $\mathrm{CH}_{3}$ & $\mathrm{CH}_{2} \mathrm{CH}_{3}$ & $\mathrm{CH}_{2} \mathrm{CH}_{3}$ & $\mathrm{CH}_{3}$ \\
\hline Q & $\mathrm{CH}_{3}$ & $\mathrm{CH}_{3}$ & $\mathrm{CH}_{3}$ & $\mathrm{CH}_{2} \mathrm{CH}_{3}$ & $\mathrm{CH}_{2} \mathrm{CH}_{3}$ & $\mathrm{CH}_{2} \mathrm{CH}_{3}$ \\
\hline B2 & $\mathrm{CH}_{3}$ & $\mathrm{CH}_{3}$ & $\mathrm{CH}_{3}$ & $\mathrm{CHCH}_{2}$ & $\mathrm{CHCH}_{2}$ & $\mathrm{CHCH}_{2}$ \\
\hline
\end{tabular}

Figure 2 Enniatins identified in methanol extract of $F$. tricinctum by (+)-electrospray liquid chromatography and mass spectrometry.

Table 1 Retention times and mass spectral data of the chromatographic peaks depicted in Figure 1 obtained from (+)-ESI LC-MS spectral analysis of the constituents of the methanol extract

$\mathrm{MS}[\mathrm{M}+\mathrm{H}]^{+}$

\begin{tabular}{lllllll} 
Peaks no. & $t_{R(\mathrm{~min})}$ & $M S(\mathrm{~m} / \mathrm{z})$ & & MS/MS $[M+H]^{+}$, (abundance) & Compounds \\
\hline 1 & 30.98 & 626.4014 & $513.3118(12.5), 413.2605(15), 300.1775(16.7), 214.1425(66.7), 196.1319(100), 86.0968(29.2)$ & 7 & Enniatin B2 \\
2 & 32.39 & 676.4148 & $551.3115(9.2), 451.2985(7.7), 326.1615(19.2), 226.1099(50), 208.0994(100), 98.0638(16.9)$ & 10 & Enniatin Q \\
3 & 32.54 & 640.4171 & $527.3296(11.5), 427.2781(9.2), 314.1748(15.4), 214.1425(60), 196.1326(100), 86.0971(15.4)$ & 7 & Enniatin B \\
4 & 33.83 & 654.4329 & $541.3446(9.2), 441.2930(7.8), 314.1943(10), 214.1425(53.8), 196.1324(100), 86.0971(11.5)$ & 7 & Enniatin B1 \\
5 & 34.99 & 668.4488 & $541.3484(8.4), 441.2957(5.7), 328.2117(16.4), 228.1588(48.4), 210.1486(100), 100.1125(31.3)$ & 7 & Enniatin A1 \\
6 & 36.04 & 682.4642 & $555.3606(10.2), 455.3087(7.8), 328.2101(11.7), 228.1585(44.5), 210.1480(100), 100.1124(11.7)$ & 7 & Enniatin A \\
\hline
\end{tabular}

Abbreviations: DBE, double bond equivalent; MS; mass spectrometry; MS/MS, tandem mass spectrometry. 

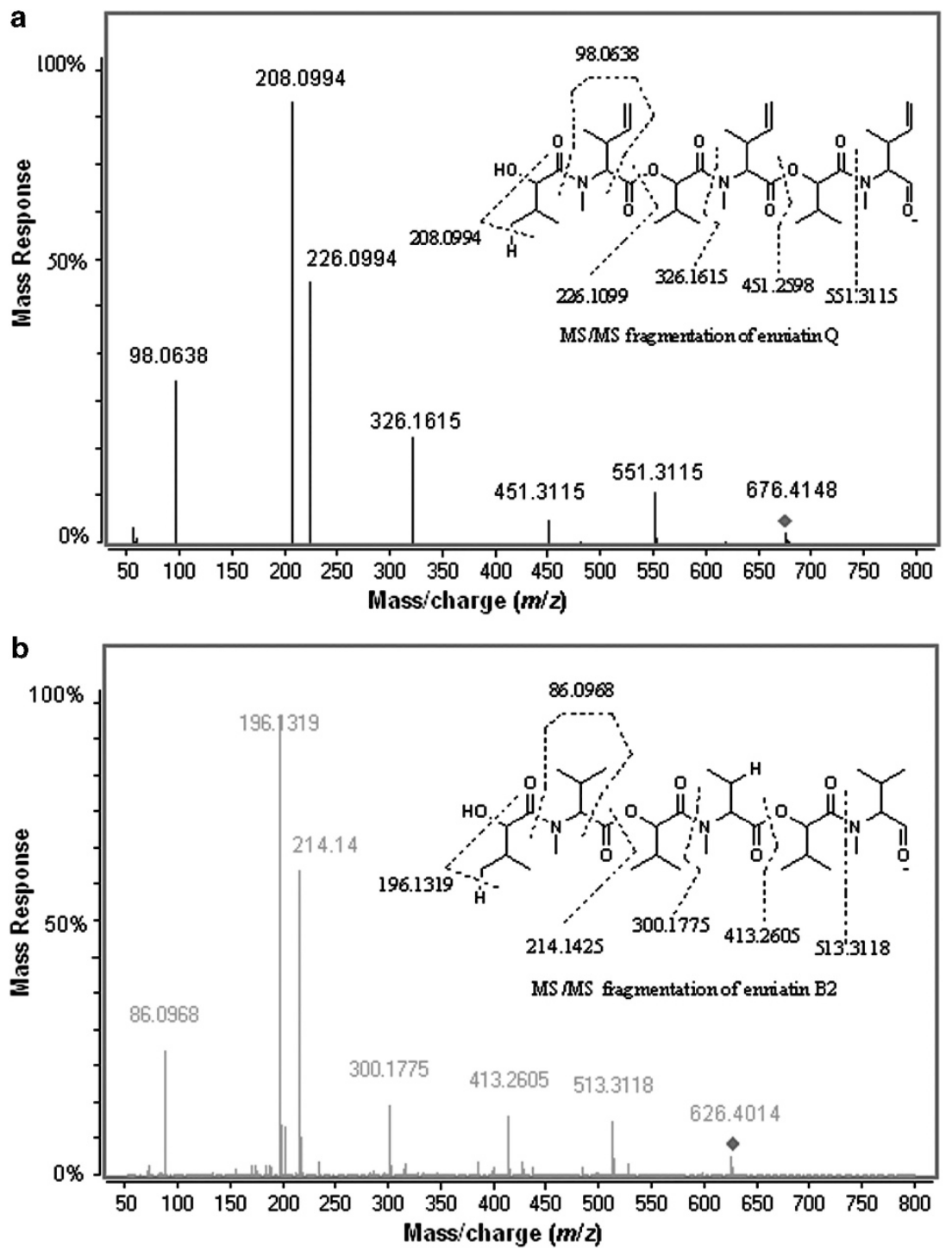

Figure 3 Tandem mass spectrometry fragmentation of enniatins $Q$ (a) and B2 (b) by (+)-electrospray liquid chromatography and tandem mass spectrometry. A full color version of this figure is available at The Journal of Antibiotics journal online.

\section{ACKNOWLEDGEMENTS}

Authors are indebted to the Joint Supervision Program of Egypt 2013-2014 for financial support to AM Zaher. We are thankful to Mr Richard Davis and Dr Peter Panizzi from the Department of Drug Discovery and Development at the Harrison School of Pharmacy, Auburn University for retesting the F. tricinctum extract against Staphylococcus aureus Xen 31.

1 Nilanonta, C. et al. Unusual enniatins produced by the insect pathogenic fungus Verticillium hemipterigenum: isolation and studies on precursor-directed biosynthesis Tetrahedron 59, 1015-1020 (2003).

2 Cordero, A., Pearce, C. J., Oberlis, N. H. Revisiting the enniatins: a review of their isolation, biosynthesis, structure determination and biological activities. J. Antibiot. 65 541-549 (2012).

3 Zhukhlistova, N. E., Tishchenko, G. N., Tolstykh, I. V., Zenkova, V. A. Molecular and crystal structure of the antibiotic enniatin $\mathrm{B}$, a secondaty microbial metabolite. Crystallogr. Rep. 44, 8-12 (1999).

4 Gupta, S., Montllor, C., Hwang, Y. S. Isolation of novel beauvericin analogues from the fungus Beauveria bassiana. J. Nat. Prod. 58, 733-738 (1995).

5 Hyun, U., Lee, D. H., Lee, C., Shin, C. G. Apoptosis induced by enniatins H and MK1688 isolated from Fusarium oxysporum FB1501. Toxicon 53, 723-728 (2009).

6 Logrieco, A., Rizzo, A., Ferracane, R., Ritieni, A. Occurrence of beauvericin and enniatins in wheat affected by Fusarium avenaceum head blight. Appl. Environ. Microbiol. 68, 82-85 (2002).
7 Tomoda, $\mathrm{H}$. et al. Inhibition of acyl-CoA: cholesterol acyltransferase activity by cyclodepsipeptide antibiotics. J. Antibiot. 45, 1626-1632 (1992).

8 Shemyakin, M. M., Ovchinnikov, Y. U., Ivanov, V. T., Kiryushkin, A. A. The structure of enniatins and related antibiotics. Tetrahedron 19, 581-591 (1963).

9 Herrmann, M., Zocher, R., Haese, A. Enniatin production by Fusarium strains and its effect on potato tuber tissue. Appl. Environ. Microbiol. 62, 393-398 (1996).

$10 \mathrm{Lin}, \mathrm{Y}$. et al. A novel compound enniatin $\mathrm{G}$ from the mangrove fungus Halosarpheia sp. from the South China Sea. Austr. J. Chem. 55, 225-227 (2002).

11 Song, H. H., Lee, H. S., Lee, G. P., Ha, S. D., Lee, C. Structural analysis of enniatin H I, and MK1688 and beauvericin by liquid chromatography-tandem mass spectrometry (LC-MS/MS) and their production by Fusarium oxysporum KFCC 11363P. Food Addit. Contam 26, 518-526 (2009).

12 Meca, G. et al. Isolation and purification of enniatins A, A1, B, B1, produced by Fusarium tricinctum in solid culture, and cytotoxicity effects on Caco-2 cells. Toxicon 56, 418-424 (2010).

13 Jain, M. et al. Synthesis, antimalarial, antileishmanial, and antimicrobial activities of some 8-quinolinamine analogues. Bioorg. Med. Chem. 13, 4458-4466 (2005).

14 Bauer, A. W., Kirby, W. M., Sherris, J. C., Turck, M. Antibiotic susceptibility testing by a standardized single disk method. Amer. J. Clin. Pathol. 45, 493-496 (1966).

15 Munigunti, R., Calderón, A. I. Development of liquid chromatography/mass spectrometry based screening assay for PfTrxR inhibitors using relative quantitation of intact thioredoxin. Rap. Commun. Mass Spectrom. 26, 2051-2056 (2012).

16 Uhlig, S., Jestoi, M., Knutsen, A. K., Heier, B. T. Multiple regression analysis as a tool for the identification of relations between semi-quantitative LC-MS data and cytotoxicity of extracts of the fungus Fusarium avenaceum (syn. F. arthrosporioides). Toxicon 48, 567-579 (2006). 